\title{
Tumefactive Demyelinating Lesions in Patients with Relapsing Remitting Multiple Sclerosis Treated with Fingolimod
}

\author{
Rocco Totaro*, Caterina Di Carmine and Antonio Carolei
}

Multiple Sclerosis Center, Department of Neurology, University of L'Aquila, Italy

\begin{abstract}
We report a case of a woman with relapsing remitting multiple sclerosis presenting with tumefactive demyelination at disease onset and treated with natalizumab after failure of first-line drugs. Patient was shifted from natalizumab to fingolimod treatment because of progressive multifocal leukoencephalopathy risk. Thirteen months after starting fingolimod treatment she developed a relapse with brain MRI findings of tumefactive demyelinating lesions. We also present a literature review on similar cases discussing a possible role of the drugs in determining brain tumefactive lesions.
\end{abstract}

Keywords: Multiple sclerosis; Fingolimod; Natalizumab; Tumefactive lesion

\section{Introduction}

Multiple sclerosis (MS) is an inflammatory-demyelinating disease of the central nervous system (CNS). Treatment options have been changing during the last two decades with novel emerging agents acting at different levels of the immune system. These drugs proved effective in preventing relapses and disability progression. Natalizumab (NAT) and fingolimod (FTY720) are the most widely used agents when firstline drugs therapy fail to control disease activity in relapsing remitting (RR) MS patients.

NAT is a humanized monoclonal antibody that blocks alpha4-beta1 integrin-mediated leukocytes migration in the CNS. NAT significantly reduces relapse rate, lesion accumulation and disability progression in RRMS [1,2]. However, long duration therapy increases progressive multifocal leukoencephalopathy (PML) risk, prompting clinicians to switch to other medications, especially in the so-called triple-riskpatients, i.e. positive JC virus antibody status, NAT treatment $>2$ years, and prior immunosuppressive treatment [3].

FTY720 is a sphingosine 1-phosphate receptor modulator that binds to lymphocytes preventing their egress from peripheral lymphoid tissues into the blood stream. FTY720 is the first oral drug approved for the treatment of RRMS and significantly reduces relapse rate, lesion accumulation and disability progression in these patients [4,5].

FTY720 widespread use in patients discontinuing NAT because of PML risk led to accumulating experience about its efficacy, safety and tolerability [6]. Recently, published cases described the occurrence of tumefactive demyelination (TD) in patients switched to FTY720 after previous treatment with first or second line agents [7-12].

We discuss a case of a woman who experienced TD after switching to FTY720 following NAT discontinuation because of PML risk.

\section{Case Report}

A 25-year old female patient was referred to our clinic on November 2005 complaining of vertigo, dizziness, and dysarthria. Magnetic resonance imaging (MRI) showed multiple T2weighted hyperintensities and a large enhancing demyelinated lesion consistent with TD. The cerebrospinal fluid examination showed positive oligoclonal bands and she was diagnosed with RRMS. Expanded disability status scale (EDSS) score evaluated at that time was 2.5.

On October 2006 she started treatment with glatiramer acetate, which was stopped on January 2007 because of occurrence of new clinical relapses. Thus, she started interferon $\beta$-1a ( $44 \mathrm{mcg}$ three time a week) until October 2008 when she switched to NAT because of a clinical and radiological disease reactivations. EDSS calculated at that time was 3.5. NAT treatment was carried out until December 2011 when she was switched to FTY720 treatment because of PML risk due to presence of anti JC virus antibody and 34 months of NAT treatment. During this treatment period no relapses or MRI activity were observed and EDSS score remained stable at 3.5.

On January 2013, after 13 months of treatment with FTY720, she presented with rapid onset right-sided sensory motor hemi syndrome, aphasia, and psychomotor agitation. Brain MRI revealed a left-sided, large, subcortical, fronto-temporo-parietal lesion with vasogenic oedema, consistent with TD. An additional subcortical lesion in the right frontal lobe, with the same signal characteristics, was also found. Gadolinium administration was not done because of a previous allergic reaction. The patient discontinued FTY720 and underwent a short pulse steroid therapy (1g IV methylprednisolone for 5 days) with partial recovery. MRI was repeated 1 month later revealing additional new T2 weighted hyper intensities and persistence of the lesion in the left hemisphere. All aforementioned lesions were enhancing after gadolinium infusion, with an open ring appearance of the left frontotemporo-parietal lesion.

NAT treatment was restarted after a six weeks washout period and subsequent follow-up showed no clinical relapses or new MRI lesions. EDSS score at the restart of NAT was 4.5, returning to the score of 3.5 in the successive months.

\section{Discussion}

Tumefactive demyelination, per se, is an uncommon manifestation

*Corresponding author: Rocco Totaro, Multiple Sclerosis Center, Department of Neurology, University of L'Aquila, Via Vetoio, 167100 L'Aquila, Italy, Tel: +39.0862.368322; Fax: +39.0862.368322; E-mail: totaro@cc.univaq.it

Received November 26, 2013; Accepted February 07, 2014; Published February 17,2014

Citation: Totaro R, Di Carmine C, Carolei A (2014) Tumefactive Demyelinating Lesions in Patients with Relapsing Remitting Multiple Sclerosis Treated with Fingolimod. J Neurol Neurophysiol S12: 006. doi:10.4172/2155-9562.S12-006

Copyright: $\odot 2014$ Totaro R, et al. This is an open-access article distributed under the terms of the Creative Commons Attribution License, which permits unrestricted use, distribution, and reproduction in any medium, provided the original author and source are credited. 
Citation: Totaro R, Di Carmine C, Carolei A (2014) Tumefactive Demyelinating Lesions in Patients with Relapsing Remitting Multiple Sclerosis Treated with Fingolimod. J Neurol Neurophysiol S12: 006. doi:10.4172/2155-9562.S12-006

of inflammatory-demyelinating diseases of the CNS [13]. If present, it is more likely to occur in the very early stage of disease and recurrences are not frequent [14]. Possible association between FTY720 and TD comes from several case reports describing the occurrence of TD in patients under FTY720 treatment [7-12]. Clinical and radiological characteristics of these cases were quite heterogeneous, with one patient being finally diagnosed with inflammatory disorder other than MS [8]. Four patients had been treated with immunomodulatory drugs (interferon $\beta-1 \mathrm{a}$, interferon $\beta-1 \mathrm{~b}$, glatiramer acetate) before FTY720 $[8,9,11,12]$, while in one case FTY720 was initiated after NAT discontinuation [10]. FTY720 treatment was started after a mean washout period of 11 weeks (range, 7-16weeks). TD occurred after a mean time lapse of 9.4 weeks after FTY720 start (range, 2-20 weeks). Therapeutic decision of FTY720 interruption occurred in two cases $[8,11]$. No case presented with TD at onset, but in a recent case report TD recurred in a patient after a first tumefactive relapse while on FTY720 treatment [7].

Compared with previous reports, our case differs since the patient suffered a TD at disease onset, suggesting a possible individual susceptibility to TD recurrence. The patient was switched to FTY720 after two immunomodulatory drugs and 34-months therapy with NAT. It is worth noting that TD occurred 13 months after FTY720 treatment, showing the longest time to relapse compared with previous reports.

FTY720 exerts an action upon the immune system not yet fully understood but it may be associated with an immunological dysregulation leading to an imbalance of T-cells within the CNS. In a recent published case there was a predominance of effector memory $\mathrm{T}$ cells (CD45R0posCCR7neg) in the peripheral blood with a decrease of CD8 effectors (CD45R0negCCR7-neg) [7]. Surprisingly, the amount of CD8 effectors in the CNS was disproportionally increased, disclosing an imbalance not exhibited in other FTY720 treated patients. A history of TD associated with autoantibody mediated inflammation and a permissive lymphocyte subset profile might suggest a potential risk condition in FTY720 treatment decision paradigm.

Because of the paucity of available data, a possible association of FTY720 and TD is still speculative. Further studies are warranted to sort this issue out and clarify FTY720 unique mechanism of action upon the immune system.

\section{References}

1. Polman $\mathrm{CH}, \mathrm{O}$ 'Connor PW, Havrdova E, Hutchinson M, Kappos L et al. (2006) A randomized, placebo-controlled trial of natalizumab for relapsing multiple sclerosis. N Engl J Med 354: 899-910.

2. Rudick RA, Stuart WH, Calabresi PA, Confavreux C, Galetta SL, et al. (2006) Natalizumab plus interferon beta-1a for relapsing multiple sclerosis. $\mathrm{N}$ Engl $\mathrm{J}$ Med 354: 911-923.

3. Havla J, Kleiter I, Kümpfel T (2013) Bridging, switching or drug holidays - how to treat a patient who stops natalizumab? Ther Clin Risk Manag 9: 361-369.

4. O'Connor P, Comi G, Montalban X, Antel J, Radue EW, et al. (2009) Oral fingolimod (FTY720) in multiple sclerosis: two-year results of a phase II extension study. Neurology 72: 73-79.

5. Kappos L, Radue EW, O'Connor P, Polman C, Hohlfeld R, et al. (2010) A placebo-controlled trial of oral fingolimod in relapsing multiple sclerosis. $\mathrm{N}$ Engl J Med 362: 387-401.

6. Bourdette D, Gilden D (2012) Fingolimod and multiple sclerosis: four cautionary tales. Neurology 79: 1942-1943.

7. Pilz G, Harrer A, Wipfler P, Oppermann K, Sellner J, et al. (2013) Tumefactive MS lesions under fingolimod: a case report and literature review. Neurology 81: $1654-1658$.

8. Min JH, Kim BJ, Lee KH (2012) Development of extensive brain lesions following fingolimod (FTY720) treatment in a patient with neuromyelitis optica spectrum disorder. Mult Scler 18: 113-115.

9. Visser F, Wattjes MP, Pouwels PJ, Linssen WH, van Oosten BW (2012) Tumefactive multiple sclerosis lesions under fingolimod treatment. Neurology 79: $2000-2003$.

10. Jander S, Turowski B, Kieseier BC, Hartung HP (2012) Emerging tumefactive multiple sclerosis after switching therapy from natalizumab to fingolimod. Mult Scler 18: 1650-1652.

11. Castrop F, Kowarik MC, Albrecht H, Krause M, Haslinger B, et al. (2012) Severe multiple sclerosis relapse under fingolimod therapy: incident or coincidence? Neurology 78: 928-930.

12. Daelman L, Maitrot A, Maarouf A, Chaunu MP, Papeix C, et al. (2012) Severe multiple sclerosis reactivation under fingolimod 3 months after natalizumab withdrawal. Mult Scler 18: 1647-1649.

13. Hardy TA, Chataway J (2013) Tumefactive demyelination: an approach to diagnosis and management. J Neurol Neurosurg Psychiatry 84: 1047-1053.

14. Altintas A, Petek B, Isik N, Terzi M, Bolukbasi F, et al. (2012) Clinical and radiological characteristics of tumefactive demyelinating lesions: follow-up study. Mult Scler 18: 1448-1453. 